\title{
Simulation of Gas-Liquid Mixture Movement through a Pipeline on the Seabed, Taking into Account the Heat Exchange Process
}

\author{
Elkhan M. Abbasov' ${ }^{1}$, Gulshan R. Agayeva1, Nurlana A. Agayeva², Tarana S. Kengerli² \\ ${ }^{1}$ Institute of Mathematics and Mechanics of NAS of Azerbaijan, Baku, Azerbaijan \\ ${ }^{2}$ Oil and Gas Research and Design Institute, SOCAR, Azerbaijan, Baku, Azerbaijan \\ Email: aelhan@mail.ru
}

How to cite this paper: Abbasov, E.M., Agayeva, G.R., Agayeva, N.A. and Kengerli, T.S. (2019) Simulation of Gas-Liquid Mixture Movement through a Pipeline on the Seabed, Taking into Account the Heat Exchange Process. Journal of Applied Mathematics and Physics, 7, 3073-3082. https://doi.org/10.4236/jamp.2019.712216

Received: September 18, 2019

Accepted: December 15, 2019

Published: December 18, 2019

Copyright $\odot 2019$ by author(s) and Scientific Research Publishing Inc. This work is licensed under the Creative Commons Attribution International License (CC BY 4.0).

http://creativecommons.org/licenses/by/4.0/

\begin{abstract}
In the paper, a dynamical model of gas-liquid mixture motion through a pipeline with regard to change of rheological properties that occur as a result of heat-exchange process, is constructed, and the solutions of the obtained connected differential equations are given. Analytic expression allowing to determine pressure change along the length of a pipeline, is obtained.
\end{abstract}

\section{Keywords}

Equations, Mathematical Model, Gas-Liquid System, Heat-Exchange Process, Rheological Properties

\section{Introduction}

In most cases, in offshore fields, transportation of produced hydrocarbons is carried out along the seabed. With such transportation, an intensive heat exchange process takes place between the environment and gas-liquid mixture.

Currently, the issues related to non-stationary head exchange and the movement of oil and gases in underground pipelines, remain insufficiently studied, and this is associated with the complexity of the thermal and hydrodynamic processes occurring in the system oil pipeline-environment. In a theoretical study of the problem, the greatest difficulties arise due to the need of simultaneous accounting of such factors as complexity of the considered rheological parameters, strong temperature dependence of rheological parameters, need of accounting mutual thermal influence of oil pipeline and external environment, i.e. to consider the thermal process as a conjugate one [1].

A great majority of papers on the laminar motion of liquid with variable 
rheological properties are connected with the study of heat exchange and motion of non-Newtonian liquid in the stationary mode (Bussel-Gretz problem and some of its generalizations). In the classical assumptions of the latter, among which there is an assumption on constancy of physico-mechanical properties of liquid, the problem for the case of pumped flow of viscoplastic and many other non-Newtonian media, received its completed solution in the papers of Sellars, Traibus, Klein, A.K. Mirzajanzade, Z.P. Shulman, E.L. Smorodinskii, G.B. Freustater and other researchers. In view of relative complexity of the obtained solutions, that in the given case [2]-[13] is associated first of all with "anomaly" of the rheological properties, in future searches were made for finding simplifying methods for approximate solution of the problem and limits for their applicability was established. For the first time, the problem of steady heat exchange during the motion of viscous liquid with regard to temperature dependence of the viscosity factor under first kind boundary conditions on the pipe wall, was considered by L.S. Leibenzon [14]. The first approximate analytic solution of this problem obtained under the assumption that temperature and viscosity of liquid are constant over the cross section and change only along the length of the pipe, belongs to him. A further study of non-Newtonian liquids whose rheological parameters change due to temperature change, transportation of these fluids on very large scales contributed to the emergence of a number of studies of heat exchange problems under laminary motion in pipes. The solution of the problem with regard to temperature dependence of rheological characteristics meets great mathematical difficulties due to nonlinearity of the initial system of energy and motion equations and necessity of joint solution of these equations. In this connection, the known analytic solutions were obtained as a rule by approximate methods and under serious assumptions. R.M. Sattarov and R.M. Mamedov offered approximate solution of the Nusselt-Gretz problem in a plane channel for a nonlinear-viscoplastic medium whose behavior was described by Kesson's generalized equation with regard to dependence of plastic viscosity and ultimate shear stress on temperature [5].

Significant successes in the study of the problem of steady heat exchange when non-Newtonian media move in channels with regard to heat dependence of rheological parameters were achieved owing to the use of numerical methods. The latter ones have great advantage before analytical methods, they allow to avoid many simplifications and achieve significant commonality of results. Some quality and quantity estimations of the influence of variability of rheological properties on heat exchange and motion are given based on analysis of numerical solutions. The essential influence on specific form of temperature dependence of rheological parameters on termohydrodynamical process, is shown.

In spite of numerous investigations, the study of gas-liquid mixture motion through a pipeline on the seabed with regard to profile and thermal dependence of rheological properties, remains poorly studied and urgent. Therefore, simulation of gas-liquid mixture motion through a pipeline on the seabed with regard to heat exchange and pipeline profile has both important practical and scientific 
value and this problem is devoted to this issue.

In the paper, we construct a model of gas-liquid mixture motion through a pipeline on the seabed with regard to heat exchange process and develop a technique for solving the obtained related differential equations.

\section{Problem Statement}

Let us consider gas-liquid mixture motion in a pipeline. To this end we partition the pipeline into three parts (Figure 1):

- The first part is the segment from the surface to the bottom of the sea;

- The second part is bottom length;

- The third part is the segment from the bottom to the sea surface.

In the first approximation, we accept mixture as homogeneous and consider the motion of homogeneous gas (or almost homogeneous).

The equation of motion of such gas is described by the Charniy equation [15]:

$$
\begin{gathered}
-\frac{\partial P}{\partial x}=\frac{\partial Q}{\partial t}+2 a Q-\rho g \\
-\frac{1}{c^{2}} \frac{\partial P}{\partial t}=\frac{\partial Q}{\partial x} .
\end{gathered}
$$

In the case when the flow is stationary, we have

$$
\frac{\partial Q}{\partial t}=0, \frac{\partial P}{\partial t}=0 \Rightarrow \frac{\partial Q}{\partial x}=0, Q=\text { const } .
$$

From the first equation of (1) we get

$$
-\frac{\mathrm{d} P}{\mathrm{~d} x}=2 a Q-\rho g .
$$

The gas density can be determined from the formula

$$
\rho=\frac{\rho_{\mathrm{atm}}}{P_{\mathrm{atm}}} \frac{T_{0}}{T} P .
$$

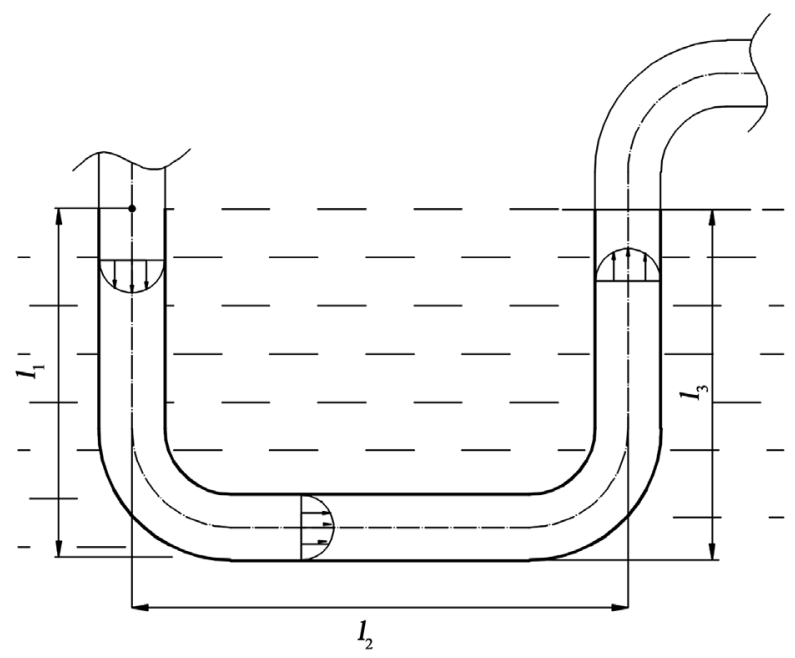

Figure 1. Pipeline profile. 
Substituting expression (3) in Equation (2), we get

$$
-\frac{\mathrm{d} P}{\mathrm{~d} x}=2 a Q-\frac{\rho_{\mathrm{atm}}}{P_{\mathrm{atm}}} \frac{T_{0}}{T} P g,
$$

or

$$
\frac{\mathrm{d} P}{\mathrm{~d} x}-\frac{\rho_{\mathrm{atm}}}{P_{\mathrm{atm}}} \frac{T_{\mathrm{atm}}}{T} P g=-2 a Q, Q=\text { const } .
$$

Here $P$ is mixture pressure at any cross-section, $P_{\text {atm }}$ is atmospheric pressure, $\rho_{\text {atm }}$ is mixture's density under atmospheric pressure, $g$ is the free fall acceleration, $T$ is mixture's temperature in any cross-section, $T_{\text {atm }}$ is mixture's temperature under atmospheric pressure, $Q$ is consumption, $a$ is resistance factor.

Accepting the external medium temperature change along the water thickness as linear, we get (Figure 2).

$$
T_{1}=T_{3}-\frac{T_{3}-T_{2}}{l} x
$$

where $l$ is the length of setting of the pipeline into water, $T_{2}$ is water temperature in the seabed, $T_{3}$ is water temperature in the surface.

Taking into account only convective part of heat emission for heat transfer equation we have [16] [17] [18]:

$$
\begin{gathered}
\frac{\mathrm{d} T}{\mathrm{~d} x}=-\frac{\beta}{R}\left(T-T_{1}\right) \\
\beta=\frac{2 k_{0}}{R V^{*}\left[c_{g} \rho_{g} \varphi+c_{l} \rho_{l}(1-\varphi)\right]} .
\end{gathered}
$$

$R$ is the inner radius of the pipeline, $V^{*}$ is averaged velocity of gas flow through the piper's cross section.

$\rho_{g}$ is gas density.

$\rho_{l}$ is liquid's density, $c_{g}$ is specific heat-capacity of gas under the given pressure, $c_{l}$ is specific heat capacity of liquid, $\varphi$ is volume fraction of gas in mixture.

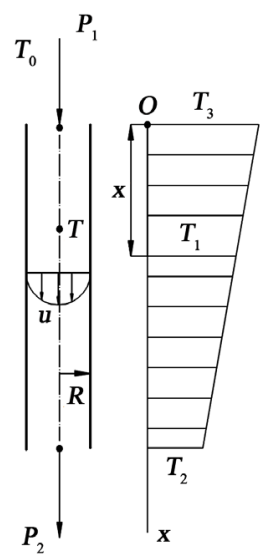

Figure 2. Design scheme. 
$k_{0}$ is heat emission between fluid and pipeline's wall.

Substituting the expression (6) in (7), we get

$$
\frac{\mathrm{d} T}{\mathrm{~d} x}+\frac{\beta}{R} T=\frac{\beta}{R}\left(T_{3}-\frac{T_{3}-T_{2}}{l} x\right) .
$$

For the boundary condition $\left.T\right|_{x=0}=T_{0}$ we get the solution of the equation in the form:

$$
T=\left(T_{0}-T_{3}\right) \exp \left(-\frac{\beta}{R} x\right)+T_{3}+\frac{R}{\beta} \frac{T_{3}-T_{2}}{l}\left(1-\exp \left(-\frac{\beta}{R} x\right)\right)-\frac{T_{3}-T_{2}}{l} x .
$$

As $\frac{\beta}{R} x<1$ even for $x=l \quad$ (limit value $x$ ), then

$$
\exp \left(-\frac{\beta}{R} x\right) \cong 1-\frac{\beta}{R} x
$$

Then

$$
T=T_{0}\left(1-\frac{T_{0}-T_{3}}{T_{0}} \frac{\beta}{R} x\right),
$$

where $T_{0}$ is temperature of gas-liquid mixture in the entry.

Substituting expression (10) in formula (5), we get

$$
\frac{\mathrm{d} P}{\mathrm{~d} x}-\frac{\rho_{\mathrm{atm}}}{P_{\text {atm }}} \frac{T_{\mathrm{atm}}}{T_{0}\left(1-\frac{T_{0}-T_{3}}{T_{0}} \frac{\beta}{R} x\right)} P g=-2 a Q .
$$

In the first approximation, we accept that dependence of kinematic viscosity of mixture on temperature is linear:

$$
v=v_{0}-\frac{T-T_{0}}{T_{0}-T_{4}}\left(v_{T}-v_{0}\right),
$$

where $T_{4}$ is the temperature of gas-liquid mixture at the end of the first area, $v_{0}$ is kinematic viscosity of mixture at temperature $T_{0}, v_{T}$ is kinematic viscosity of mixture at temperature $T_{4}$.

Substituting expression (10) in (12), we get

$$
v=v_{0}+\frac{T_{0}-T_{3}}{T_{0}-T_{4}}\left(v_{T}-v_{0}\right) \frac{\beta}{R} x .
$$

The resistance factor is determined by the formula [15]:

$$
a=\frac{16 v}{d^{2}},
$$

where $d$ is the inner diameter of the pipe.

As $\frac{T_{0}-T_{3}}{T_{0}} \frac{\beta}{R} x \ll 1$ even for $I$ (limit value of $x$ ), then

$$
\frac{1}{T_{0}\left(1-\frac{T_{0}-T_{3}}{T_{0}} \frac{\beta}{R} x\right)} \cong \frac{1}{T_{0}}\left(1+\frac{T_{0}-T_{3}}{T_{0}} \frac{\beta}{R} x\right) .
$$

Then allowing for expressions (13), (14) and (15), from Equation (11) we get 


$$
\frac{\mathrm{d} P}{\mathrm{~d} x}-M(x) P=Q(x),
$$

where

$$
\begin{gathered}
Q(x)=a_{1}+a_{2} x, M(x)=a_{0}+b_{0} x \\
a_{1}=-32 \frac{Q}{d^{2}} v_{0}, a_{2}=-32 \frac{Q}{d^{2}} \frac{T_{0}-T_{3}}{T_{0}-T_{4}}\left(v_{T}-v_{0}\right) \frac{\beta}{R} \\
a_{0}=g \frac{\rho_{\text {atm }}}{P_{\text {atm }}} \frac{T_{\text {atm }}}{T_{0}}, g \frac{\rho_{\text {atm }}}{P_{\text {atm }}} \frac{T_{\text {atm }}}{T_{0}} \frac{T_{0}-T_{3}}{T_{0}} \frac{\beta}{R}=b_{0} .
\end{gathered}
$$

The solution of Equation (16) will be of the form:

$$
P=\exp \left(\int M(x) \mathrm{d} x\right) \int Q(x) \exp \left(-\int M(x) \mathrm{d} x\right) \mathrm{d} x+c_{3} \exp \left(\int M(x) \mathrm{d} x\right),
$$

where $c_{3}$ is an integration constant.

For the values of parameters of the system entering the parameter $b_{0}$, $\frac{b_{0} x^{2}}{2}<1$.

Then in the first approximation

$$
\exp \left(\frac{b_{0} x^{2}}{2}\right) \approx 1+\frac{b_{0} x^{2}}{2} .
$$

Allowing for expressions (17) and (19), from expression (18) we get

$$
\begin{aligned}
P= & \left(1+\frac{b_{0} x^{2}}{2}\right) \exp \left(a_{0} x\right)\left(a_{1} \int \exp \left(-a_{0} x\right)\left(1-\frac{b_{0} x^{2}}{2}\right) \mathrm{d} x\right. \\
& \left.+a_{2} \int x \exp \left(-a_{0} x\right)\left(1-\frac{b_{0} x^{2}}{2}\right) \mathrm{d} x\right)+c_{3}\left(1+\frac{b_{0} x^{2}}{2}\right) \exp \left(a_{0} x\right)
\end{aligned}
$$

Having differentiated expression (20), we get

$$
\begin{aligned}
P_{2}= & \left(1+\frac{b_{0} x^{2}}{2}\right)\left(\frac{a_{1}}{a_{0}}-\frac{b_{0} a_{1}}{2 a_{0}^{3}}\left(a_{0}^{2} x^{2}+2 a_{0} x+2\right)\right. \\
& -\frac{a_{2}}{2 a_{0}^{4}}\left(a_{0}^{3} b_{0} x^{3}-3 a_{0}^{2} b_{0} x^{2}-2 a_{0}^{3} x+6 a_{0} b_{0} x+2 a_{0}^{2}-6 b_{0}\right) . \\
& +c_{3}\left(1+\frac{b_{0} x^{2}}{2}\right) \exp \left(a_{0} x\right)
\end{aligned}
$$

We find $c_{3}$ from the boundary condition $\left.P\right|_{x=0}=P_{1}$.

$$
c_{3}=P_{1}-\frac{a_{1}}{a_{0}}+\frac{b_{0} a_{1}}{a_{0}^{3}}+\frac{a_{2}}{a_{0}^{2}}-\frac{3 a_{2} b_{0}}{a_{0}^{4}} .
$$

Now we consider the horizontal part of the pipeline (Figure 3).

As in the seabed the temperature of gas-liquid mixture and environment is balanced, the heat exchange between them comes to an end. Then the equation of gas motion will be of the form:

$$
\begin{gathered}
\frac{\mathrm{d} P}{\mathrm{~d} x}=-2 a Q \\
P=P_{2}-2 a Q x \\
\left.P\right|_{x=l_{2}}=P_{3}
\end{gathered}
$$




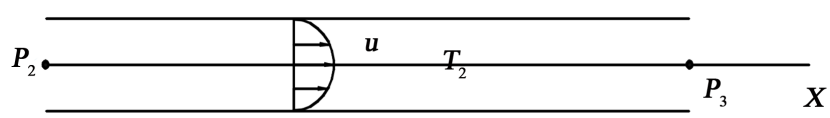

Figure 3. Design scheme.

$$
P_{3}=P_{2}-2 a Q l_{2},
$$

where $P_{2}$ is pressure at the end of the first area, $l_{2}$ is the length of the second area of the pipeline, $P_{3}$ is pressure at the end of the second area.

Similar (6) for the third area the temperature change will occur by the law (we will locate the origin of the coordinate axis $x$ in the lower section of the pipeline)

$$
T_{1}=T_{2}+\frac{T_{3}-T_{2}}{l} x
$$

The heat transfer equation similar to (7) will be in the form:

$$
\frac{\mathrm{d} T}{\mathrm{~d} x}=\frac{\beta}{R}\left(T-T_{1}\right) .
$$

Allowing for formula (26), from expression (27) we have:

$$
T=T_{2}\left(1+2 \frac{\beta}{R} x\right) \text {. }
$$

Substituting expression (28) in formula (5), we get

$$
\frac{\mathrm{d} P}{\mathrm{~d} x}+\frac{\rho_{\mathrm{atm}}}{P_{\mathrm{atm}}} \frac{T_{\mathrm{atm}}}{T_{2}}\left(1-2 \frac{\beta}{R} x\right) P g=-2 a Q .
$$

Allowing for expression (29) and linear dependence of kinematic dependence of mixture on temperature:

$$
v=v_{0}+\frac{T_{0}-T_{3}}{T_{0}-T_{2}}\left(v_{T}-v_{0}\right) .
$$

we get an equation similar to expression (16):

$$
\frac{\mathrm{d} P}{\mathrm{~d} x}+M_{1}(x) P=Q_{1}(x) \text {. }
$$

where

$$
\begin{gathered}
Q_{1}(x)=a_{3}+a_{4} x, M_{1}(x)=c_{0}+d_{0} x \\
a_{3}=-32 \frac{Q}{d^{2}} v_{0}, a_{4}=-64 \frac{Q}{d^{2}} \frac{T_{2}}{T_{3}-T_{2}}\left(v_{T}-v_{0}\right) \frac{\beta}{R} \\
c_{0}=g \frac{\rho_{\mathrm{atm}}}{P_{\mathrm{atm}}} \frac{T_{\mathrm{atm}}}{T_{2}}, d_{0}=-2 g \frac{\rho_{\mathrm{atm}}}{P_{\mathrm{atm}}} \frac{T_{\mathrm{atm}}}{T_{2}} \frac{\beta}{R} .
\end{gathered}
$$

The solution of Equation (31) has the form:

$$
\begin{aligned}
P=\exp \left(\int M_{1}(x) \mathrm{d} x\right) \int Q_{1}(x) \exp \left(-\int M_{1}(x) \mathrm{d} x\right)+c_{3} \exp \left(\int M_{1}(x) \mathrm{d} x\right) \\
P_{4}=\left(1-\frac{b_{0} x^{2}}{2}\right)\left(\frac{a_{1}}{a_{0}}+\frac{b_{0} a_{1}}{2 a_{0}^{3}}\left(a_{0}^{2} x^{2}-2 a_{0} x+2\right)\right. \\
+\frac{a_{2}}{2 a_{0}^{4}}\left(a_{0}^{3} b_{0} x^{3}-3 a_{0}^{2} b_{0} x^{2}+2 a_{0}^{3} x+6 a_{0} b_{0} x-2 a_{0}^{2}-6 b_{0}\right) \\
+c_{4}\left(1-\frac{b_{0} x^{2}}{2}\right) \exp \left(-a_{0} x\right)
\end{aligned}
$$


We find $c_{4}$ from the boundary condition $\left.P\right|_{x=0}=P_{3}$

$$
c_{4}=P_{3}-\frac{a_{3}}{c_{0}}+\frac{d_{0} a_{3}}{c_{0}^{3}}+\frac{a_{4}}{c_{0}^{2}}+\frac{3 a_{4} d_{0}}{c_{0}^{4}} .
$$

Using (34), we can find pressure at the end of the pipeline but already on the sea surface:

$$
\begin{aligned}
P_{4}= & \left(1-\frac{b_{0} l_{3}^{2}}{2}\right)\left(\frac{a_{1}}{a_{0}}+\frac{b_{0} a_{1}}{2 a_{0}^{3}}\left(a_{0}^{2} l_{3}^{2}-2 a_{0} l_{3}+2\right)\right. \\
& +\frac{a_{2}}{2 a_{0}^{4}}\left(a_{0}^{3} b_{0} l_{3}^{3}-3 a_{0}^{2} b_{0} l_{3}^{2}+2 a_{0}^{3} l_{3}+6 a_{0} b_{0} l_{3}-2 a_{0}^{2}-6 b_{0}\right) \\
& +c_{4}\left(1-\frac{b_{0} l_{3}^{2}}{2}\right) \exp \left(-a_{0} l_{3}\right) .
\end{aligned}
$$

\section{Discussion of Results}

\begin{tabular}{|c|c|}
\hline Variable & Value \\
\hline Atmospheric pressure $P_{\text {atm }}(\mathrm{Pa})$ & $10^{5}$ \\
\hline Density under atmospheric pressure $\rho_{\text {atm }}\left(\mathrm{kg} / \mathrm{m}^{3}\right)$ & 0.668 \\
\hline$v_{0}$ is kinematic viscosity of mixture at temperature $T_{0} \quad\left(\mathrm{~m}^{2} / \mathrm{s}\right)$ & $3.4 \times 10^{-5}$ \\
\hline$v_{T}$ is kinematic viscosity of mixture at temperature $T_{4} \quad\left(\mathrm{~m}^{2} / \mathrm{s}\right)$ & $1.14 \times 10^{-4}$ \\
\hline$T_{0}$ is temperature of gas-liquid mixture in the entry, $\left({ }^{\circ} \mathrm{C}\right)$ & 20 \\
\hline$T_{2}$ is water temperature in the seabed, $\left({ }^{\circ} \mathrm{C}\right)$ & 5 \\
\hline$T_{3}$ is water temperature in the surface $\left({ }^{\circ} \mathrm{C}\right)$ & 30 \\
\hline$T_{4}$ is the temperature of gas-liquid mixture at the end of the first area, $\left({ }^{\circ} \mathrm{C}\right)$ & 20 \\
\hline$T_{\text {atm }}$ atmospheric temperature $\left({ }^{\circ} \mathrm{C}\right)$ & 20 \\
\hline$a$ is resistance factor $\left(\mathrm{s}^{-1}\right)$ & 0.04 \\
\hline$g$ is the free fall acceleration $\left(\mathrm{m} / \mathrm{s}^{2}\right)$ & 9.8 \\
\hline$R$ is the inner radius of the pipeline $(\mathrm{m})$ & 0.1524 \\
\hline Q is consumption $(\mathrm{kg} / \mathrm{s})$ & 17.78 \\
\hline$l_{1}$ is the length of the first area of the pipeline, $(\mathrm{m})$ & 110 \\
\hline$l_{2}$ is the length of the second area of the pipeline, $(\mathrm{m})$ & 1000 \\
\hline$l_{3}$ is the length of the third area of the pipeline, $(\mathrm{m})$ & 107 \\
\hline$P_{1}$ is mixture pressure at the entrance $(\mathrm{Pa})$ & $1.6 \times 10^{6}$ \\
\hline
\end{tabular}

Taking into account the values of practical parameters (Table 1) from the expression (22), (26), (34) we calculate $P_{2}, P_{3}, P_{4}$.

Table 1. The values of parameters. 


$$
P_{2}=1.849 \times 10^{6} \mathrm{~Pa} ; P_{3}=1.847 \times 10^{6} \mathrm{~Pa} ; P_{4}=1.05 \times 10^{6} \mathrm{~Pa}
$$

\section{Conclusion}

Based on the carried out investigations, we obtain analytic expression allowing to determine pressure change by the length of the pipeline with regard to thermal dependence of rheological properties of gas-liquid mixture that happens as a result of heat exchange process. The results of numerical calculations at practical values of the system parameters show that a change in the rheological properties of a liquid as a result of a heat exchange process with the environment has a significant effect on pressure loss. In this case, due to a change in the rheological properties of the liquid, the pressure loss during its movement in the pipeline increases by about $25 \%$ compared with the movement of the liquid without changing them.

\section{Conflicts of Interest}

The authors declare no conflicts of interest regarding the publication of this paper.

\section{References}

[1] Khalikov, R.A. (1984) Laminary Motion of Viscoplastic Liquid in Pipes in Nonstationary Conjugate Heat Exchange Conditions. Moscow, 141 p.

[2] Kuzmin, S.I. (2005) Heat Exchange and Friction in Rheological Systems with Regard to Variable Viscosity of Fluid. Astrakhan, 160 p.

[3] Froishteter, G.B., Danilevich, S.Yu. and Radionova, N.V. (1990) Flow and Heat Exchange of Non-Newtonian Liquids in Pipes. Kiev, Naukova Dumka, 216 p.

[4] Zavyagin, V.G. and Vorotnikov, D.A. (2004) Mathematical Models of Non-Newtonian Liquids. Voronezh State University, Voronezh, 43 p.

[5] Sattarov, R.M. and Mamedov, R.M. (1988) Some Unstationary Motion of Incompressible Medium in Pipes. Az.IOCh, Baku, 63 p.

[6] Acheson, D.J. (2005) Elementary Fluid Dynamics. Oxford, 397 p.

[7] Huseynzadeh, M.A., Druchina, L.I., Petrova, O.N. and Stepanova, M.F. (1991) Hydrodynamical Processes in Complex Pipeline Systems. Nedra, Moscow, 168 p.

[8] Suleimanov, B.A., Abbasov, E.M. and Sisenbayeva, M.R. (2017) Mechanism of Gas Saturated Oil Viscosity Anomaly Near to Phase Transition Point. Physics of Fluids, 29, Article ID: 012106. https://doi.org/10.1063/1.4974081

[9] Bolotov, D.A., Mirzajanzadeh, A.Kh. and Nesterov, I.I. (1988) Rheological Properties of Gas and Liquid Mixtures in the Saturation Pressure Field. Izv. Ansssr, MzhG, No. 1.

[10] Pham, S.T., Truong, M.H. and Pham, B.T. (2017) Flow Assurance in Subsea Pipeline Design for Transportation of Petroleum Products. Open Journal of Civil Engineering, 7, 311-323. https://doi.org/10.4236/ojce.2017.72021

[11] Zheng, W.K. and Jiang, Y.Q. (2019) Distribution Characteristics of Gas-Liquid Mixture in Spiral-Wound Heat Exchanger under Sloshing Conditions. Cryogenics, 99, 68-77. https://doi.org/10.1016/j.cryogenics.2019.03.001

[12] Buleiko, V.M., Grigoryev, B.A. and Ovsyanikova, M.S. (2017) Investigation of Phase 
Behavior of Hydrocarbon Mixtures with Low Condensate Rations. News of Gas Science, 2, 4-14.

[13] Gudala, M.K., Banerjee, S., Kumar, R., Rao, T.R.M., Mandal, A. and Kumar, T. (2018) Experimental Investigation on Hydrodynamics of Two-Phase Crude Oil Flow in Horizontal Pipe with Novel Surfactant. Journal of Fluids Engineering, 140, Article ID: 061302. https://doi.org/10.1115/1.4039130

[14] Targ, S.M. (1951) Fundamental Problems of Theory of Laminary Flows. QITTL, Moscow, $400 \mathrm{p}$.

[15] Charniy, I.A. (1975) Unstationary Motion of Liquids in Pipes. Nedra, Moscow, 295 p.

[16] Faizullayev, D.F. (1966) Laminary Motion of Multiphase Media in Pipelines. FAN, Tashkent, $219 \mathrm{p}$.

[17] Abbasov, E.M. and Feizullayev, Kh.A. (2016) Mathematical Simulation of Gas Liquid Mixture Flow in the Bed and in the Pipe with Regard to Dynamical Relation of the System "Bed-Well". Computational Mathematics and Mathematical Physics, 56, 142-154.

[18] Lourie, M.V. (2012) Mathematical Simulation of Processes of Pipeline Transportation of Oil, Oil Products and Gas. Manual, 456 p. 